\title{
Identificação dos constituintes químicos da fração volátil da Calendula officinalis produzida no Paraná
}

\author{
Zilda Cristiani Gazim'; Gilberto Alves Ferreira' ${ }^{1}$; Claudia Moraes Rezende²; Celso Vataru Nakamura²; \\ Benedito Prado Dias Filho²; Diógenes Aparicio Garcia Cortez ${ }^{3}$ \\ IUNIPAR, Praça Mascarenha de Moraes s/n, 87500-000 Umuarama-PR; ${ }^{2}$ Instituto de Química, UFRJ, Centro de Tecnologia, Bloco A, \\ 21945-970 Rio de Janeiro-RJ; ${ }^{2}$ epto. de Análises Clínicas; ${ }^{3}$ Depto. de Farmácia e Farmacologia da UEM, Av. Colombo 5790, 87020 - \\ 900 Maringá-PR; E-mail: dagcortez@uem.br
}

\section{RESUMO}

A Calendula officinalis L., pertence à família Asteraceae, é originária da Europa e está aclimatada na região Sul do Brasil, onde vem crescendo o seu cultivo comercial para fins farmacêuticos e cosméticos. Suas inúmeras propriedades terapêuticas entre as quais antiinflamatória, cicatrizante e anti-séptica, justificam a grande procura desta planta pela população. O presente trabalho compara a composição química da fração volátil da cultura de calêndula aclimatada na região Noroeste do Paraná, com os dados da literatura da cultura na França. As substancias químicas majoritários identificadas por cromatografia gasosa acoplada à espectrometria de massas (CG/MS), nas duas culturas, foram sesquiterpenos hidrocarbonetos e álcoois; d-cadineno (22,53\%) e a-cadinol (20,40\%) para a cultura de calêndula produzida na região Noroeste do Paraná, Brasil, e d-cadineno $(12,10 \%)$ e a-cadinol $(25,50 \%)$ distinto do registrado para a cultura de calêndula produzida na região de Massif Central, França. Adicionalmente, para garantir a qualidade da planta na comercialização, foram realizadas análises físico-químicas (perda por secagem $=85,0 \%$ e perda por dessecação $=11,6 \%$ ).

Palavras-chave: Calendula officinalis, cultivo, óleo essencial, composição química.

\begin{abstract}
Chemical compounds of the Calendula officinalis volatile fraction produced in the Paraná State, Brazil

Calendula officinalis L., belongs to the Asteraceae family from Europe and is acclimatized in the south region of Brazil where its commercial cultivation has been increasing for pharmaceutical and cosmetic purposes. Its several therapeutic properties (antiinflammatory, healing, antiseptic) justify the great demand for this plant by the population. The present study compares the chemical composition of the volatile fraction of two cultivations of calendula, one from Massif Central, France, and the other acclimatized in the Northeast region of Paraná State, Brazil. Most compounds identified by Gas Chromatography-mass spectrometry (GC-MS) in both cultivations included the sesquiterpenes hydrocarbons and alcohols; d-cadinene $(22.53 \%)$ and á-cadinol $(20.40 \%)$ for the cultivation of the calendula produced in the Northeast region of Paraná State, and d-cadinene $(12.10 \%)$ and a-cadinol $(25.50 \%)$ for the cultivation of the calendula produced in Massif Central, France. In addition, physical-chemical analyses (determination of water at room temperature $=85.0 \%$ and loss on drying $=11.6 \%$ ), were carried out in order to assure the quality of the plant for commercialization.
\end{abstract}

Keywords: Calendula officinalis, cultivation, essential oil, chemical composition.

(Recebido para publicação em 15 de março de 2006; aceito em 26 fevereiro de 2007)

\begin{abstract}
$\mathrm{A}$ Calendula officinalis L. pertence família Asteraceae, é uma planta herbácea, nativa em toda região do Mediterrâneo e cultivada em toda a Europa, onde floresce quase o ano todo, sendo comum encontrá-la em jardins como planta orrnamental (Cromack \& Smith, 1996). Temperaturas noturnas muito elevadas diminuem o tamanho das flores (Correa Júnior et al., 1991). É muito sensível à falta de água nos períodos de estiagem, o que acarreta um comprometimento da sua produtividade (Silva Júnior, 1997); daí a necessidade de sistemas de irrigação na área de plantio.

A calendula se desenvolve melhor e é mais produtiva quando cultivada no inverno, sendo inclusive resistente a geadas leves (Montanari Júnior, 2000). Apresenta como principais constituin-
\end{abstract}

tes químicos saponinas triterpênicas (Alonso, 1998); flavonóides (Pietta et al., 1992, 1994); hidroxicumarinas (PDR, 2000); carotenóides (Stevenson, 1961; Alonso, 1998); triterpenos pentacíclicos trihidroxiálcoois (Wilkomirski, 1985); taninos; poliacetilenos; esteróis; sesquiterpenos glicosídeo (PDR, 2000); e um óleo volátil $(0,1-0,2 \%)$ muito abundante em sesquiterpenos hidrocarbonetos e álcoois (Chalchat et al., 1991; PDR, 2000; Radulescu et al., 2000; Crabas et al., 2003).

É extensamente utilizada pelas indústrias farmacêutica, cosmética e alimentícia e pela população em geral por causa das suas atividades antiinflamatória (Della Loggia et al., 1994) e antiedematosa (Hamburger et al., 2003). Observou-se que o extrato de C. officinalis induziu a formação de vasos sanguíneos. Esse resultado foi muito importante no processo de granulação (PDR, 2000; Schulz et al., 2002). Outras atividades farmacológicas tem sido reportadas tais como: imunomodulatória (Amirghofran et al., 2000) por estimulação na granulocitose; antitumoral (Elias et al., 1990); antimutagênica e antiviral (Kalvatchev et al, 1997; PDR, 2000); antimicrobiana (Dumenil et al., 1980; Gracza, 1987; Hsieh et al, 2001). Outras atividades biológicas tais como moluscicida (Helaly et al., 1999); o óleo essencial como nematicida (Perez et al., 2003); genotóxica (Ramos et al., 1998); larvicida (El-Shazly \& Hussein, 2004); e antiplasmódica (Boyom et al., 2003). 
Na perfumaria, é utilizado na composição de vários perfumes. O aroma característico das flores de calêndula é associado a presença dos sesquiterpenos na fração volátil, dentre esses o d-cadineno que confere as notas verdes, doces e refrescantes; e o a-cadinol responsável pelas notas amadeiradas (Reznicek \& Zitterl-Eglseer, 2003).

O objetivo deste trabalho foi comparar a composição química do óleo essencial extraído das folhas da C. officinalis cultivada na região Noroeste do Paraná comparado com os dados da composição química do óleo essencial extraído desta planta na região de Massif Central França.

\section{MATERIAL E MÉTODOS}

A espécie foi identificada pela Prof ${ }^{a}$. Dr $^{\mathrm{a}}$. Ezilda Jacomassi da Universidade Paranaense, e, uma exsicata dessa espécie foi depositada no Herbário dessa Universidade.

O solo da amostra 1, usado no presente experimento apresentou as características químicas: $\mathrm{pH}$ em $\mathrm{CaCl}_{2}=4,8$; $\mathrm{pH}$ em $\mathrm{H}_{2} \mathrm{O}=5,7 ; \mathrm{Al}^{+++}=0,00 \mathrm{cmol} \mathrm{dm}_{\mathrm{c}}^{-3}$; $\mathrm{H}^{+}+\mathrm{Al}^{+++}=2,94 \quad \mathrm{cmol}_{\mathrm{c}} \mathrm{dm}^{-3}$; $\mathrm{Ca}^{++}+\mathrm{Mg}^{++}=2,84 \mathrm{cmol} \mathrm{dm}_{\mathrm{c}}^{-3} ; \mathrm{Ca}^{++}=1,82$ $\mathrm{cmol}_{\mathrm{c}} \mathrm{dm}^{-3} ; \quad \mathrm{K}^{+}=0,14 \quad \mathrm{cmol}_{\mathrm{c}} \mathrm{dm}^{-3}$; $\mathrm{cmol}_{\mathrm{c}} \mathrm{dm}^{-3} ; \mathrm{P}=19 \mathrm{mg} \mathrm{dm}^{-3}$ e $\mathrm{C}=6,59 \mathrm{mg}$ $\mathrm{dm}^{-3}$. Na amostra 2 as características químicas foram: $\mathrm{pH}$ em $\mathrm{CaCl}_{2}=5,6 ; \mathrm{pH}$ em $\mathrm{H}_{2} \mathrm{O}=6,5 ; \quad \mathrm{Al}^{+++}=0,00 \quad \mathrm{cmol}_{\mathrm{c}} \mathrm{dm}^{-3}$; $\mathrm{H}^{+}+\mathrm{Al}^{+++}=2,54 \quad \mathrm{cmol}_{\mathrm{c}}^{\mathrm{c}} \mathrm{dm}^{-3}$; $\mathrm{Ca}^{++}+\mathrm{Mg}^{++}=4,08 \mathrm{cmol} \mathrm{dm}_{\mathrm{c}}^{-3} ; \mathrm{Ca}^{++}=3,18$ $\mathrm{cmol}_{\mathrm{c}} \mathrm{dm}^{-3} ; \quad \mathrm{K}^{+}=0,71 \quad \mathrm{cmol}_{\mathrm{c}} \mathrm{dm}^{-3}$; $\mathrm{cmol}_{\mathrm{c}} \mathrm{dm}^{-3} ; \mathrm{P}=49 \mathrm{mg} \mathrm{dm}^{-3}$ e $\mathrm{C}=7,75 \mathrm{mg}$ $\mathrm{dm}^{-3}$.

A implantação da cultura da $C$. officinalis foi realizado em um terreno de $34,5 \mathrm{~m}^{2}$ localizado no Horto Medicinal da Universidade Paranaense-UNIPAR, em Umuarama, região Noroeste do Paraná, nas coordenadas $S 23^{\circ} 46,225^{\prime}$ e WO $53^{\circ}$ 16,730' e altitude de $391 \mathrm{~m}$.

As amostras de solo para análise foram coletadas em duas partes do terreno, a uma profundidade de $20 \mathrm{~cm} \mathrm{em}$ diferentes pontos, resultando em duas amostras compostas (amostras 1 e 2). A análise do solo foi realizada no laboratório da UEM. A calagem foi realizada conforme resultado da análise química do solo. Os valores de $\mathrm{pH}$ e as concentrações dos elementos químicos encontrados no solo estão nas Tabelas 1 e 2.

Tabela 1. Substâncias químicas identificadas na fração volátil de Calendula officinalis (Chemical substances identified in the volatile fraction of Calendula officinalis).

\begin{tabular}{llccl}
\hline $\mathbf{N}^{\text {o }}$ & Substância & Area $\%$ & IRR calc. & IRR lit. \\
\hline 1 & $\alpha$-copaeno & 0,96 & 1365 & 1376 \\
2 & $\alpha$-ionona & 1,54 & 1421 & 1426 \\
3 & $\alpha$-humuleno & 1,18 & 1444 & 1454 \\
4 & Geranilacetona & 1,61 & 1452 & 1453 \\
5 & $\gamma$-muuroleno & 2,27 & 1472 & 1477 \\
6 & $\beta$-ionona & 3,25 & 1484 & 1485 \\
7 & Ledeno & 2,30 & 1488 & 1493 \\
8 & $\alpha$-muuroleno & 5,62 & 1497 & 1499 \\
9 & $\gamma$-cadineno & 8,93 & 1511 & 1513 \\
10 & $\delta$-cadineno & 22,53 & 1522 & 1524 \\
11 & $\alpha$-cadineno & 0,92 & 1533 & 1538 \\
12 & $\alpha$-calacoreno & 2,32 & 1539 & 1542 \\
13 & Óxido de cariofileno & 0,52 & 1547 & 1581 \\
14 & Copaen-4- -ol & 0,64 & 1566 & 1584 \\
15 & b-oplopenona & 1,73 & 1568 & 1606 \\
16 & Viridiflorol & 2,25 & 1585 & 1590 \\
17 & Ledol & 1,30 & 1595 & 1565 \\
18 & 1,10 -di-epi-Cubenol & 0,87 & 1608 & 1614 \\
19 & 1-epi-cubenol & 1,60 & 1621 & 1627 \\
20 & epi $\alpha$-muurolol & 12,87 & 1639 & 1641 \\
21 & $\alpha$-cadinol & 20,40 & 1654 & 1653 \\
22 & Cadaleno & 0,76 & 1671 & 1674 \\
\hline $1 R R$ & & & &
\end{tabular}

IRR calc__Índice de retenção relativo calculado em coluna capilar DB5 ( 5\% fenilmetilsiloxane). IRR lit.-_́ndice de retenção relativo encontrado na literatura em coluna capilar DB5. EM- Comparação com Espectros de Massas da espectroteca Wiley 275. Lit.Comparação dos Índices de Retenção e/ou dos Espectros de Massas com a Literatura (Adams, 1995) $($ IRRcalc $=$ Relative retention index, obtained in a capilar column DB5 (5\% of phemylmethylsiloxane) IRRlit= Relative retention index obtained in a capilar column DB5. $\mathrm{EM}=$ Comparison to mass spectra from the Wiley 275 library. Lit= Comparison of the retention indexes and/or from the mass spectra obtained from literature (Adams, 1995)).

O plantio ocorreu em 30/04/04 (início do outono), e coincidiu com temperaturas mais amenas. As mudas foram obtidas a partir de sementes da variedade Orange Guitana de cor cinza (Fiallo et al., 2000) e foram obtidas da empresa Sementes Semex. O plantio foi realizado em sistema de semeadura direta no campo em linha (Montanari Júnior, 2000) e em canteiros, que é recomendado para as espécies de ciclo curto e porte pequeno a médio, como a C. officinalis (Correa Júnior et al., 1991). As covas foram abertas nos canteiros com espaçamento entre linhas de 0,40 m e na linha de $0,30 \mathrm{~m}$, sendo plantadas três sementes/cova com profundidade de $2 \mathrm{~cm}$ (Montanari Júnior, 2000). Foi utilizado adubo orgânico (esterco de gado curtido) na dosagem de 2 $\mathrm{kg} / \mathrm{m}^{2}$. O calcário e o adubo orgânico foram misturados ao solo em uma profundidade de $20 \mathrm{~cm}$, permanecendo por uma semana descansando antes de iniciar o plantio. Por se tratar de uma cultura totalmente orgânica, foi utilizado para o combate de pragas, macerado de fumo (10 $\mathrm{cm}$ de fumo de corda em um litro de água) pulverizado sobre as plantas. A aplicação foi realizada quatro vezes até o controle total das pragas (Correa Júnior et al., 1991).

A coleta foi iniciada em 20/07/04, três meses após o plantio, quando as flores estavam no seu desenvolvimento máximo com indícios de polinização (Oliveira et al., 1998). A coleta das flores foi diariamente no período da manhã (7 h 30 min) e ao entardecer (18 h) (Simões et al., 2001). As coletas não foram realizadas em dias chuvosos e tomou-se cuidado para esperar que todo o orvalho evaporasse antes da coleta. As flores foram colhidas manualmente, com o pedúnculo o mais curto possível (Montanari Júnior, 2000). 


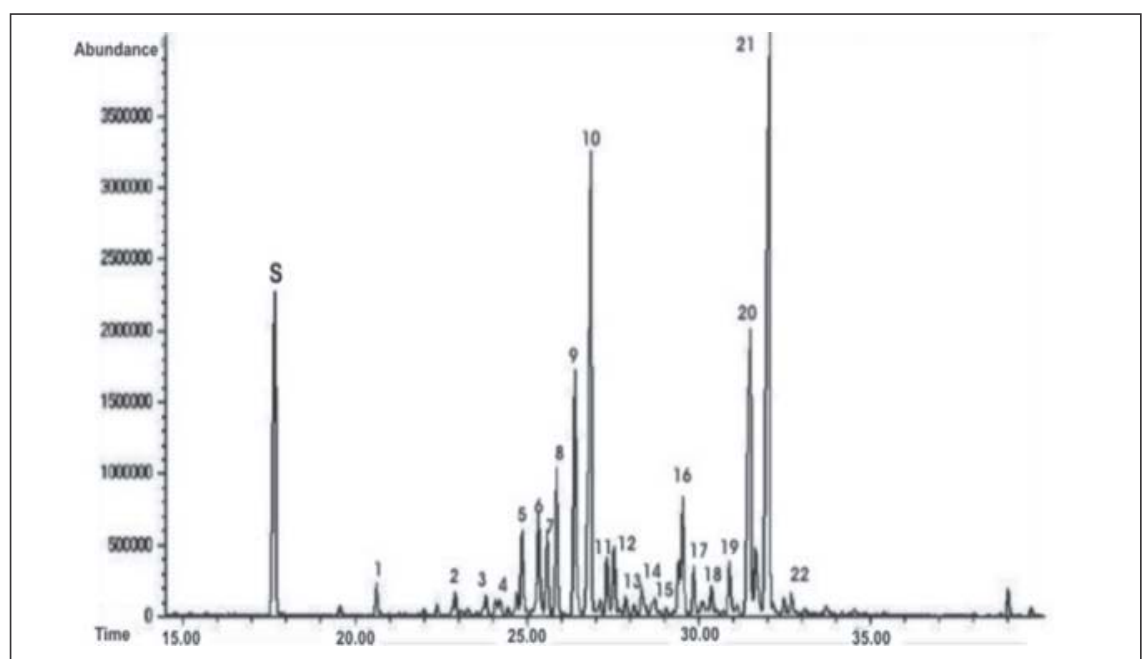

Figura 1: Cromatograma da fração volátil obtida por arraste a vapor da C. officinalis cultivada na região Noroeste do Paraná, Brasil (chromatogram of the volatile fraction, obtained through steam distillation from $C$. officinalis cultivated in the northeastern region of Parana State).

A secagem das flores foi realizada em esteiras à sombra e em temperatura ambiente $\left(25^{\circ} \mathrm{C}\right)$. As flores foram acondicionadas em camadas finas e durante a secagem não foram revolvidas por um período de 10 dias. Nesse intervalo de secagem, foram determinados a perda de água por secagem e perda de água por dessecação.

A perda por secagem foi determinada em cinco amostras com cerca de 27 $\mathrm{g}$ das flores frescas, colocadas para secar em temperatura ambiente $\left(25^{\circ} \mathrm{C}\right)$ com ausência de luminosidade. Foram pesados diariamente até peso constante. Os resultados foram expressos em perda de substâncias voláteis e/ou água percentual, por meio da média de cinco determinações (Farmacopéia Brasileira, 1988).

A perda por dessecação foi determinada em $2 \mathrm{~g}$ das flores secas, em pesafiltro de vidro previamente dessecado e pesado nas mesmas condições a serem empregadas na determinação. Os pesafiltros foram colocados na estufa por 2 horas em $105^{\circ} \mathrm{C}$, após foi resfriado em dessecador e pesado. A operação foi repetida até peso constante. Os valores representam a média de 5 determinações e são expressos em \% (p/p) (Farmacopéia Brasileira, 1988).

A técnica utilizada para a extração da fração volátil da $C$. officinalis foi destilação por arraste a vapor com coobação em aparelho tipo Clevenger modificado (Chalchat et al., 1991; Radulescu et al., 2000). As lígulas secas, foram separadas do receptáculo floral, pesadas $50 \mathrm{~g}$ de planta e adicionados $500 \mathrm{ml}$ de água destilada. Em seguida, foi procedida a análise. A análise cromatográfica foi realizada no Instituto de Química da UFRR. A identificação dos constituintes químicos do óleo essencial de $C$. officinalis foi realizada por cromatografia gasosa de alta resolução com detector de massas computadorizado CGAR-EM. Para a análise cromatográfica, foi utilizado um cromatógrafo Agilent 6890 Seies II acoplado a um espectrômetro de massas Agilent 5973. Condições do detector: ionização por impacto de elétrons $70 \mathrm{eV}$, interface $280^{\circ} \mathrm{C}$, linha de transferência $280^{\circ} \mathrm{C}$ e fonte iônica $230^{\circ} \mathrm{C}$. Condições de operação no cromatógrafo: injetor no modo "splitless" (tempo de válvula 0,5 min) em $250^{\circ} \mathrm{C}$; gás de arraste He com fluxo de $1 \mathrm{~mL} / \mathrm{min}$; programação de aquecimento do forno cromatográfico: $\mathrm{T}_{\text {inicial }} 35^{\circ} \mathrm{C}$ a $60^{\circ} \mathrm{C}\left(1^{\circ} \mathrm{C} \mathrm{m^{-1 }}\right), 200^{\circ} \mathrm{C}$ $\left(8^{\circ} \mathrm{C} \mathrm{min}^{-1}\right)$, e $280^{\circ} \mathrm{C}\left(15^{\circ} \mathrm{C} \mathrm{min}^{-1}\right)$, permanecendo por $5 \mathrm{~min}$. Coluna capilar DB-5 (J\&W):30 m x $0.25 \mathrm{~mm} \times 0.25$ $\mu \mathrm{m}$. Foi adicionado como padrão interno o tridecano $(\mathrm{S})$ numa concentração de $5 \%$. As estruturas foram determinadas com base na análise e na comparação de seus espectros de massas com a espectroteca Wiley 275, bem como pela com a literatura (Adams, 1995). comparação de seus índices de retenção

\section{RESULTADOS E DISCUSSÃO}

A perda de água por secagem variou de 27,010 a 27,310 g (média de 27,105 g) para as plantas frescas e de 4,023 $\mathrm{g}$ a 4,105 g (média de 4,059 g para as plantas secas). A determinação da perda por dessecação das flores de C. officinalis variou de 19,8904 a 25,2429 g (média de $22,087 \mathrm{~g}$ ou 11,6\%). Os resultados encontrados nos testes de perda de água por secagem e de perda de água por dessecação mostraram que a secagem das flores está de acordo com a literatura, pois o teor de umidade nas flores frescas varia entre $60 \%$ a $95 \%$, e o teor de umidade permitido nas flores secas deve permanecer entre 8 a $15 \%$ (Oliveira et al., 1998). Os cuidados durante as etapas de secagem do material vegetal, devem ser considerados, pois o excesso de água remanescente no vegetal, pode favorecer a proliferação microbiana e alterar os constituintes químicos, favorecendo a ação de enzimas autolíticas presentes no vegetal e, dessa forma, alterar substâncias que possam apresentar alguma atividade biológica.

Por meio da análise cromatográfica da fração volátil da cultura de $C$. officinalis produzida na região Noroeste do Paraná, foram identificadas 22 substâncias químicas (Figura 1), sendo essas pertencentes à classe dos sesquiterpenos e classificadas como sesquiterpenos hidrocarbonetos e álcoois.

Vinte e dois constituintes voláteis da C. officinalis, foram indentificados (Tabela 1) com a área que estes ocupam no cromatograma e o cálculo do Índice de Retenção Relativo (IRR). Observa-se a presença de sesquiterpenos hidrocarbonetos (1 a 13 e 22) e álcoois (14 a 21). Dentre estes, destacam-se, $\delta$ cadineno $(22,53 \%)$ e $\alpha$-cadinol $(20,40 \%)$ como constituintes majoritários presentes na fração volátil da $C$. officinalis.

A calêndula utilizada no experimento realizado por Chalchat et al. (1991) foi cultivada na região de Massif Central, França. A técnica de extração da fração volátil foi por destilação por arraste a vapor. As substâncias químicas majoritárias identificadas por CG/EM foram $\delta$-cadineno $(12,10 \%)$ e $\alpha$-cadinol $(25,50 \%)$. 
A Calendula officinalis cultivada na região Noroeste do Paraná, onde foi aclimatada, mostrou possuir semelhanças com a mesma produzida em seu habitat natural no que diz respeito à composição química da fração volátil quando comparada com dados da literatura obtido pela técnica de arraste a vapor. Conclui-se que oferecendo à planta condições ideais de plantio, de coleta, de secagem e de extração, é possível obter fração volátil com constituintes químicos semelhantes àqueles obtidos em seu habitat natural.

\section{REFERÊNCIAS}

ADAMS RP. 1995. Identification of essential oil components by gas chromatography / mass spectroscopy. Illinois: Allured Publishing Corporation. 467 p.

ALONSO JR. 1998. Tratado de fitomedicina: bases clínicas y farmacológicas. Buenos Aires: Isis Ediciones, p. 327-330.

AMIRGHOFRAN Z; AZADBAKHT M; KARIMI MH. 2000. Evaluation of the immunomodulatory effects of five herbal Journal of Ethnopharmacology, 72: 167-172.

BOYOM FF; NGOUANA V; ZOLLO PHA; MENUTC; BESSIERE JM; GUT J;

CHALCHAT JC; GARRY RPH; MICHET A 1991. Chemical Composition of Essential Oil of Calendula officinalis L. (Pot Marigold). Flavour and Fragrance Journal, 6: 189-192.

CORREA JÚNIOR C; MING LC; SCHEFFER MC. 1991. Cultivo de plantas medicinais condimentares e aromáticas. Emater, 162 p.

CRABAS N; MARONGIU B; PIRAS A; PIVETTA T; PORCEDDA S. 2003. Extraction, separation and isolation of volatiles and dyes from Calendula alis L. And Aloysia triphylla (L'Her). Britton by supercritical CO2. Journal of Essential Oil Research, 15: 350355.

CROMACK HTH; SMITH JM. 1996. Calendula officinalis- production potential and crop agronomy in southern England. Journal Industrial Crops and Products, 7: 223-229.
DELLA LOGGIA R; TUBARO A; SOSA S; BECKER H; SAAR ST; ISAAC D. 1994. The role of triperpenoids in the topical antiinflamatory activity of Calendula officinalis flowers. Planta medica, 60: 516520.

DUMENIL G; CHEMLI R; BALANSAR G; GUIRAUD H; LALLEMAND M. 1980. Étude des propriétés antibactériennes des fleurs de Souci Calendula officinalis $L$. et des teintures mères homéopathiques de $C$. officinalis $L$. et $C$. arvensis $L$. Annales Pharmaceutiques Françaises, 38 : 493-494.

ELIAS R; DE MÉO M; OLLIVIER VE; LAGET M; BALANSARD G; DUMENIL G. 1990 Antimutagenic activity of some saponins isolated from Calendula officinalis L., C. arvensis L., and Hedera helix L. Mutagenesis, 5: 327-331.

EL-SHAZLY AM; HUSSEIN KT. 2004. Chemical analysis and biological activities of the essential oil of Teucrium leucocladum Boiss. (Lamiaceae). Biochemical Systematics and Ecology, 32: 665-674.

FARMACOPÉIA BRASILEIRA. 4. ed. São Paulo: Atheneu, 1988.

FIALLO VRF; HÉRNANDEZ CLM; REYES M; JORRIN GM; BORREGO JCA; FERRADÁ CAR. 2000. Comparación entre 2 cultivares de Calendula officinalis L.. Revista Cubana de Plantas Medicinales, 5: 14-16.

GRACZA L. 1987. Oxygen-Containing Terpene Derivatives from Calendula officinalis. Planta Medica, 53: 227.

HAMBURGER M; ADLER S; BAUMANN D; FÖRG A; WEINREICH B. 2003. Preparative purification of the major anti-inflamatory triterpenoid esters from Marigold (Calendula officinalis). Fitoterapia, 74: 328-338.

HELALY FM; AHMED AA; EL-GHAFFAR MAA. 1999. Natural rubber base matrix containing Calendula officinalis plant as a source of molluscicidal saponin. Journal of Controlled Release, 57: 1-7.

HSIEH PC; MAU JL; HUANG SH. 2001. Antimicrobial effects of various combinations of plant extracts. Food Microbiology, 18: 3543.

KALVATCHEV Z; WALDER R; GARZARO D. 1997. Anti-HIV activity of extracts from Calendula officinalis flowers. Biomedical Pharmacotheraphy, 51: 176-180.
MONTANARI JÚNIOR I. 2000. Aspéctos do Cultivo Comercial de Calêndula. Revista Agroecologica Hoje, II: 24-25.

OLIVEIRA F; AKISUE G; AKISUE MK. 1998. Farmacognosia. 1.ed. Rio de Janeiro: Atheneu, 1998. p. 5-111.

PDR FOR HERBAL MEDICINE. 2000. 2nd ed. New Jersey: Montvale, p. 497-99.

PEREZ MP; NAVAS-CORTES JA; PASCUALVILLALOBOS MJ; CASTILLO P. 2003. Nematicidal activity of essential oils and organic amendments from Asteraceae against root-knot nematodes. Plant Pathology, 52: 395-401.

PIETTAP; BRUNO A; MAURI P; RAVAA. 1992. Separation of flavonol-2-O-glycosides from Calendula officinalis and Sambucus nigra by high-performance liquid and micellar electrokinetic capillary chromatography. Journal of Chromatography, 593: 165-170.

PIETTA P; FACINO RM; CARINI M; MAURI P. 1994. Thermospray liquid chromatographymass spectrometry of flavonol glycosides from medicinal plants. Journal of Chromatography A, 661: 121-126.

RADULESCU V; DONEANU C; LOLOIU T. 2000. GC Investigation of chemical composition of Calendula officinalis . Revue Roumaine de Chimie, 45: 271-275.

RAMOS A; EDREIRA A; VIZOSO A; BETANCOURT J; LÓPEZ M; DÉCALO M. 1988. Genotoxicity of an extract of Calendula officinalis L. Journal of Ethnopharmacology, 61: 49-55.

REZNICEK G; ZITTERL-EGLSEER K. 2003. Quantitative determination of the faradiol esters in marigold flowers and extracts. Scientia Pharmaceutica, 71: 121-128.

SCHULS V; HANSEL R; TYLER VE. 2002. Fitoterapia racional: um guia de fitoterapia para as ciências da saúde. São Paulo: Ed. Manole. 316 p.

SILVA JÚNIOR AA. 1997. Plantas medicinais e Aromáticas CD-ROM: Ed. Epagri S.A.

SIMÕES CMO; SCHENKEL EP; GOSMANN G; MELLO JCP; MENTZ LA; PETROWICK PR. 2001. Farmacognosia da Planta ao Medicamento, Porto Alegre: UFRGS. 821 p.

STEVENSON R. 1961. Some constituents of Calendula officinalis. Helvetica Chimica Acta, 31: 1815.

WILKOMIRSKI, B. 1985. Pentacyclic triterpene triols from Calendula officinalis flowers. Phytochemistry, 24: 3066-3067. 